\title{
Flow Behaviour of Inhaled Fibres - Equations of Motion and Preliminary Results of Real Trajectories Recorded by a High-Speed Camera
}

\author{
Frantisek Lizal ${ }^{1, *}$, Miloslav Belka ${ }^{1}$, Milan Maly ${ }^{1}$, Matous Cabalka ${ }^{2}$, Jan Jedelsky $^{1}$, and \\ Miroslav Jicha ${ }^{1}$ \\ ${ }^{1}$ Brno University of Technology, Faculty of Mechanical Engineering, Energy Institute, Technicka \\ 2896/2 Brno, 616 60, the Czech Republic \\ ${ }^{2}$ Brno University of Technology, Faculty of Mechanical Engineering, Institute of Mathematics, \\ Technicka 2896/2 Brno, 616 60, the Czech Republic
}

\begin{abstract}
The ability to precisely predict the fate of inhaled fibres is important for toxicologists as well as for pharmaceutists struggling to utilize fibres as carriers of a medication. However, the complexity of fibre movement in human airways still represents a significant challenge for programmers of codes for simulation of fibre flow. This conference contribution introduces the theoretical equations of fibre motion which can be used for calculation of the fate of inhaled fibres, and also, in the second part, first results of high-speed camera recorded trajectories of fibres downstream of a realistic human airway bifurcation are presented as an illustration of the real behaviour of fibres in the lungs.
\end{abstract}

\section{Introduction}

Inhaled fibres may give rise to various lung diseases depending on their physical and chemical properties. In general, fibrotic or malignant changes may appear as a result of long-term exposure to fibres. The crucial parameter is biopersistence. It is defined as the ability of the inhaled fibre to resist the clearance mechanisms in human airways. There are four main clearance mechanisms [1]: 1) The mucociliary escalator - acting mostly in the nose and tracheobronchial airways. It utilizes the ciliated cells covered with mucus, which catch and transport the deposited fibres using the rhythmical beating of the cilia towards the throat from where they are swallowed and subsequently expelled from the body. 2) Phagocytosis (a process by which certain living cells ingest or engulf foreign particles) by alveolar macrophages. It has been recognized, that the fibres longer than $17 \mu \mathrm{m}$ are the most dangerous [2]. The shorter fibres are usually removed, however, failure of macrophages to remove the longer fibres (frustrated phagocytosis) leads to the production of reactive oxygen species. As a result, significant damage to cell structure appears [3]. 3) Dissolution - this effect depends on the $\mathrm{pH}$ in a specific location. Fibres from various materials have different solubility. It seems wise to produce such man-made mineral fibres which will be soluble by the fluids in the human lungs and hence will be less harmful. 4) Translocation. Fibres can

*Corresponding author: lizal@ffme.vutbr.cz 
migrate across the alveolar wall to the interstitium and eventually, reach the lymphatic system [1]. Regrettably, this mechanism may induce inflammatory reaction with cytokines release, that can lead to mesothelioma [4].

It should be noted, that the ability of fibres to penetrate deep into the human lungs, could, in principle, be also used for delivery of active pharmaceutical ingredients. The idea is not new. It has been presented several times, let us mention at least the pioneers of this area, Chan and Gonda [5], who prepared fibres from cromoglycic acid for potential use in the pharmaceutical industry.

Nonetheless, regardless of the motivation, whether it comes from the toxicological community as the requirement to assess the harmful effects of a certain concentration of fibres, or from the pharmaceutical industry in order to predict the dose of a drug delivered by fibrous carrier, it is necessary to be able to calculate the fibre motion and predict the deposition sites of the inhaled fibres.

This contribution is meant as in introduction to the theoretical equations of fibre motion which can be used for calculation of the fate of inhaled fibres, and also, in the second part, first results of high-speed camera recorded trajectories of fibres downstream of a realistic human airway bifurcation are presented as an illustration of the real behaviour of fibres in the lungs.

\section{Equations of fibre motion}

The fibre motion can be decomposed into translational and rotational motion, governed by equations (1) and (2), respectively, according to Tian and Ahmadi [6]:

$$
\begin{gathered}
m^{p} \frac{d \mathbf{v}}{d t}=m^{p} \mathbf{g}+\mathbf{f}^{h}+\mathbf{f}^{L} . \\
I_{\hat{\chi}} \frac{d \omega_{\hat{x}}}{d t}-\omega_{\hat{y}} \omega_{\hat{z}}\left(I_{\hat{y}}-I_{\hat{z}}\right)=T_{\hat{x}}^{h},
\end{gathered}
$$

where $v$ is the fibre translational velocity vector, $\left(\omega_{\hat{x}}, \omega_{\hat{y}}, \omega_{\hat{z}}\right)$ are the fibre angular velocity components, $m^{p}$ denotes the mass of the fibre, $g$ is the gravitational acceleration, $\boldsymbol{f}^{\boldsymbol{h}}$ stands for the hydrodynamic drag, and $f^{L}$ is the shear-induced lift force. $\left(l_{\hat{x}}, l_{\hat{y}}, l_{\hat{z}}\right)$ are the moments of inertia about the fibre principle axes, and $\left(T_{\hat{x}}^{h}, T_{\hat{y}}^{h}, T_{\hat{z}}^{h}\right)$ are the hydrodynamic torques acting on the fibre. The hydrodynamic $\operatorname{drag} \boldsymbol{f}^{\boldsymbol{h}}$ acting on the fibre is given by:

$$
\mathbf{f}^{h}=\mu \pi a \hat{\hat{\mathbf{K}}}(\mathbf{u}-\mathbf{v})
$$

where $\mu$ is the fluid dynamic viscosity, $a$ is the semi-minor axis of the ellipsoid of revolution, $\boldsymbol{u}$ is the vector of the fluid velocity, and $\widehat{K}$ is the translational dyadic - the elements of this matrix can be calculated according to [7].

\section{Trajectories of real fibres}

The model fibres used for the recording of real trajectories were prepared of the glass wool Supafil ${ }^{\circledR}$ Loft (Knauf Insulation GmbH, Simbach am Inn, Germany), normally used for thermal insulation. The fibres with an average diameter of $3.8 \pm 1.4 \mu \mathrm{m}$ and the length of $34.1 \pm 19.0 \mu \mathrm{m}$ were prepared by the disintegration of long glass wool strings in a mechanical press. These fibres were homogenously mixed with glass beads (Ballotini 
impact glass beads, Potters Industries Inc.) to facilitate deagglomeration and dispersion of fibres.

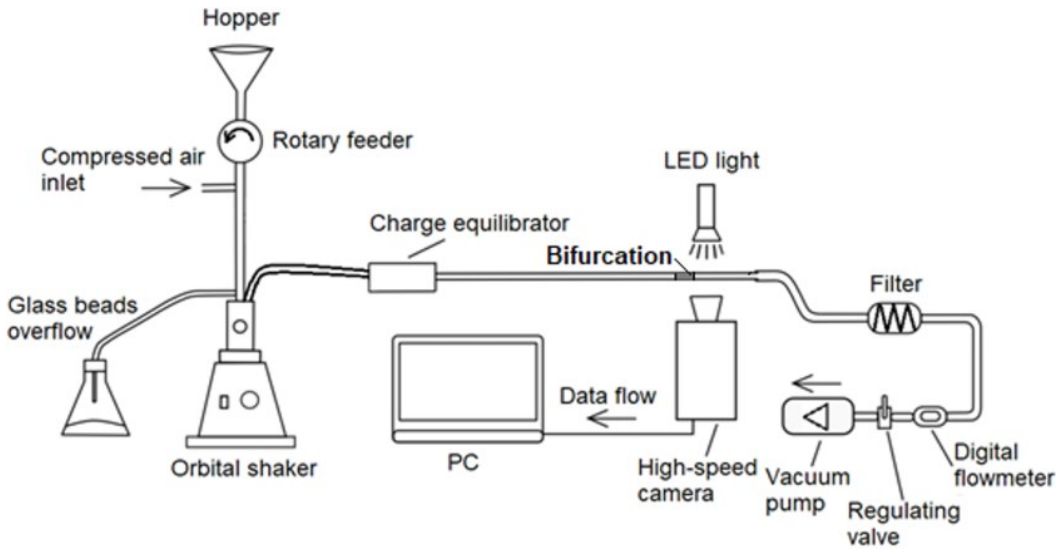

Fig. 1. A scheme of the experimental rig.

The experimental rig can be seen in Figure 1. The mixture of fibres and beads had been inserted into the hopper. The rotary feeder continuously delivered a small amount of the mixture to the fluidized bed generator based on the orbital shaker Wisd NM-10 (Witeg Labortechnik GmbH, Wertheim, Germany) with the attached in-house fluidized chamber. Electric charge equilibrator NEKR-10 (Eckert \& Ziegler CESIO, Prague, the Czech Republic) using ${ }^{85} \mathrm{Kr}$ was used to create the Boltzman equilibrium of charges. Upstream of the realistic first bifurcation of human airways fabricated by rapid prototyping was $1.5 \mathrm{~m}$ long glass tube with a diameter of $15 \mathrm{~mm}$ to ensure the developed flow. The inlet flow rate of $24 \mathrm{~L} / \mathrm{min}$, which corresponds to the flow Reynolds number of 2300, was produced by the Busch PA0008C vacuum pump (Busch Vakuum s.r.o., Brno, the Czech Republic). The recordings of fibre trajectories were performed by the Photron SA-Z high-speed camera. The resolution of images captured through a long-range microscope lens (12X Zoom, NAVITAR, New York, USA) was $1.5 \mu \mathrm{m}$ per pixel. A LED light HPLS-36DD18B (Lightspeed Technologies, USA) was used for illumination. The measurements were performed $10 \mathrm{~mm}$ downstream of the bifurcation in both daughter branches. Only results for the frontal plane visualization are presented here. The recordings were processed by an inhouse software Fiber Analyzer and Matlab.
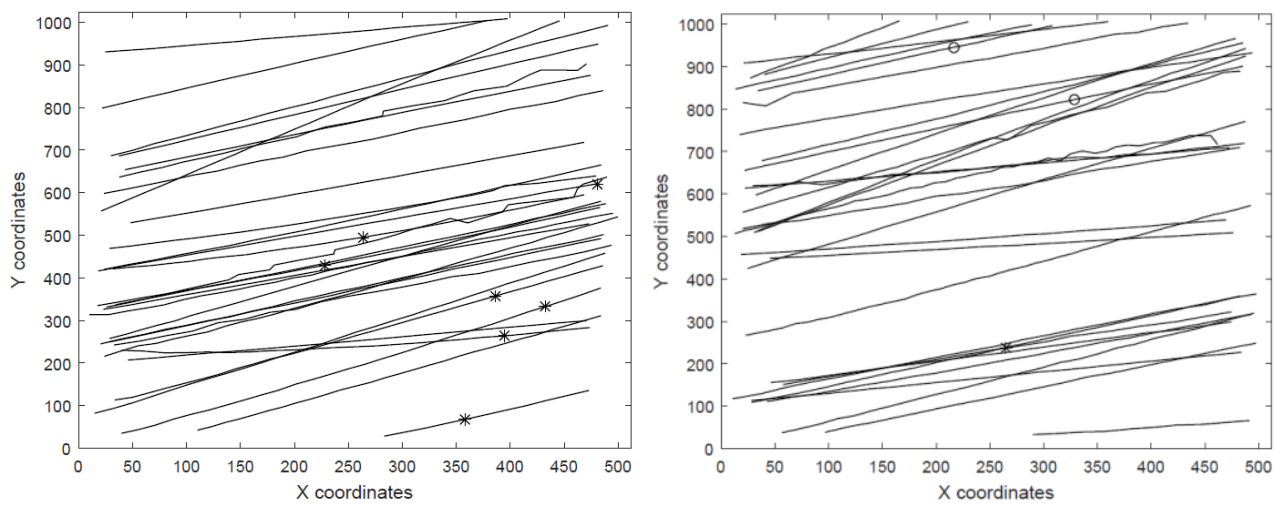

Fig. 2. The trajectories of fibres in the central axis of the left main bronchus for the flow rate of $24 \mathrm{~L} / \mathrm{min}$. The asterisks indicate flips of fibres from the orientation perpendicular to streamlines to parallel, while the circles indicate the opposite case. Each panel displays all trajectories recorded during one run of the experiment (each case was measured twice). 

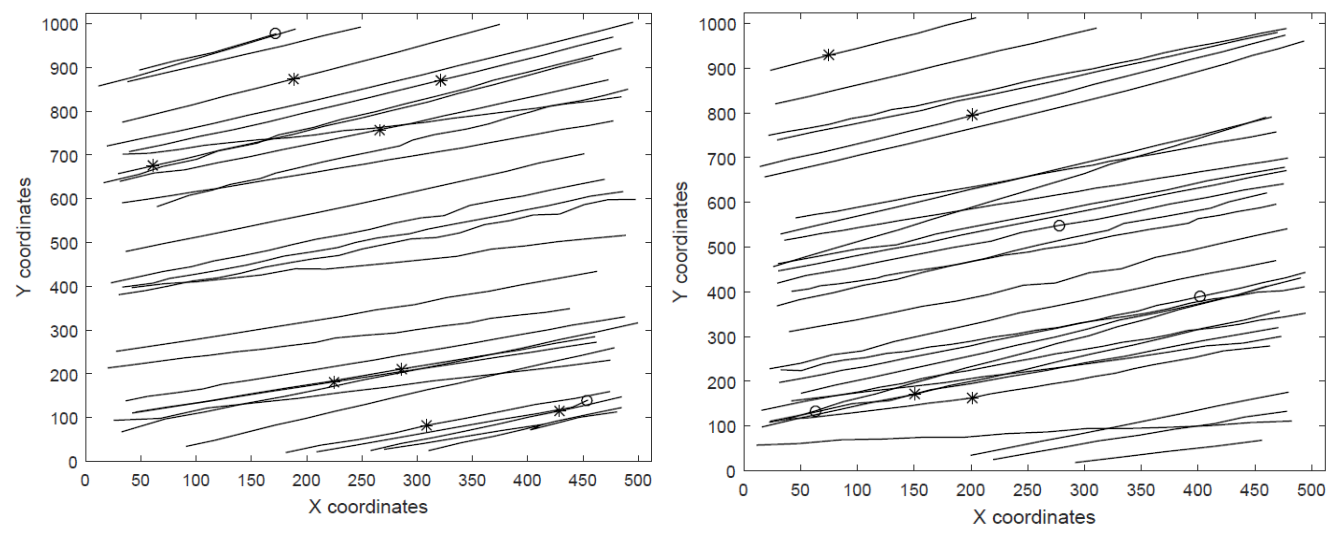

Fig. 3. The trajectories of fibres in the central axis of the right main bronchus for the flow rate of $24 \mathrm{~L} / \mathrm{min}$. The asterisks indicate flips of fibres from the orientation perpendicular to streamlines to parallel, while the circles indicate the opposite case. Each panel displays all trajectories recorded during one run of the experiment (each case was measured twice).

\section{Conclusions}

The visualization and trajectories extracted from the recordings (Figure 2 and 3 ) revealed that although the depth of field was very narrow (approx. $0.1 \mathrm{~mm}$ ) and hence all fibres flew in one plane, the fibres followed several differently inclined trajectories. Only fibres in either parallel or perpendicular orientation with respect to the streamlines were observed. Importantly, flips from parallel to perpendicular (denoted by circles in Figure 2 and 3) or from perpendicular to parallel (asterisks) orientation were recorded. Significantly more flips were observed in the right main bronchus and apparently, the flips from perpendicular to parallel orientation prevailed.

Our findings are important in the light of the widely spread notion, that fibres penetrate deep into lungs due to their ability to align with the streamlines. The results recorded during our experiments are being processed by statistical methods and a more detailed analysis of fibre behaviour in human airways will be presented soon.

The acknowledgement: This work was supported by the Czech Science Foundation under the grant GA18-25618S.

\section{References}

1. V. C. Sanchez, J. R. Pietruska, N. R. Miselis, R. H. Hurt, and A. B. Kane, Wires Nanomed Nanobi 1 (5), 511 (2009)

2. R. P. Musselman, W. C. Miiller, W. Eastes, J. G. Hadley, O. Kamstrup, P. Thevenaz, and T. W. Hesterberg, Environmental Health Perspectives 102, 139 (1994)

3. B. T. Mossman, P. J. Borm, V. Castranova, D. L. Costa, K. Donaldson, and S. R. Kleeberger, Particle and fibre toxicology 4, 4 (2007)

4. J. Fleury Feith and M. C. Jaurand, Rev Pneumol Clin 69 (6), 358 (2013)

5. H. K. Chan and I. Gonda, Journal of Aerosol Science 20 (2), 157 (1989)

6. L. Tian and G. Ahmadi, Journal of Aerosol Science 60, 1 (2013)

7. G. B. Jeffery, Proceedings of the Royal Society of London A: Mathematical, Physical and Engineering Sciences 102 (715), 161 (1922) 